\title{
Poly(ADP-ribose) polymerase 1/2 inhibitors decrease the ubiquitination of ALC1 mediated by CHFR in breast cancer
}

\author{
YIRAN WANG $^{1 *}$, YING WANG ${ }^{1 *}$, NING WANG ${ }^{1}$, LIFANG YOU $^{2}$, FEI LONG ${ }^{1}$, \\ CHANGJUAN SHAO ${ }^{2}$, YAJIE WANG ${ }^{1}$ and JIAXUE $\mathrm{WU}^{3}$ \\ ${ }^{1}$ Department of Oncology, Changhai Hospital, Second Military Medical University, Shanghai 200433; \\ ${ }^{2}$ School of Life Sciences, Fudan University, Shanghai 200438; ${ }^{3}$ Zhongshan Hospital and \\ School of Life Sciences, Fudan University, Shanghai 200032, P.R. China
}

Received March 27, 2018; Accepted June 11, 2019

DOI: $10.3892 / o r .2019 .7242$

\begin{abstract}
With the increasing use of poly(ADP-ribose) polymerase (PARP) inhibitors in cancer therapy, understanding their resistance is an urgent research quest. Additionally, CHFR is an E3 ubiquitin ligase, recruited to double-strand breaks (DSBs) by PAR. Furthermore, $A L C l$ is a new oncogene involved in the invasion and metastasis of breast cancer. Moreover, PARylated PARP1 activates ALC1 at sites of DNA damage, yet the underlying mechanism remains unclear. Mass spectrometric analysis, western blot analysis and immunoprecipitation were performed to confirm the interaction between CHFR and ALC1 in the physiological condition. Deletion mutants of CHFR and ALC1 were generated to map the interaction domain. PARP1/2 inhibitors were added to identify the ubiquitination of ALC1 by CHFR. ALC1
\end{abstract}

Correspondence to: Dr Yajie Wang, Department of Oncology, Changhai Hospital, Second Military Medical University, 168 Changhai Road, Yangpu, Shanghai 200433, P.R. China

E-mail: wangyjscholar@sina.com

Dr Jiaxue Wu, Zhongshan Hospital and School of Life Sciences, Fudan University, 2005 Songhu Road, Yangpu, Shanghai 200032, P.R. China

E-mail: jiaxue@fudan.edu.cn

${ }^{*}$ Contributed equally

Abbreviations: PARP, poly(ADP-ribose) polymerase; CHFR, checkpoint with forehead-associated and RING finger domains; ALC1, amplified in liver cancer protein 1; CHD1L, chromodomain-helicase-DNA-binding protein 1-like; UB, ubiquitin; PAR, poly-ADP-ribose; $\mathrm{CHX}$, cycloheximide; BRCA, breast cancer susceptibility gene; PBS, phosphate-buffered saline; PVDF, polyvinylidene fluoride; DMSO, dimethyl sulphoxide; ATP, adenosine triphosphate; PMD, PAR modification domain; FHA, filamentous hemagglutinin; PBZ, poly(ADP-ribose)-binding zinc finger; DSB, double strand break; HR, homologous recombination

Key words: CHFR, ALC1, breast cancer, PARP1/2 inhibitors, ubiquitination half-life was examined to compare the expression of ALC1 protein in the presence and absence of PARP1/2 inhibitors. The results revealed that the transcriptional level of ALC1 was not upregulated in breast cancer tissues. CHFR interacted with ALC1. The PBZ domain of CHFR, the PMD domain and the MACRO domain of ALC1 domain are the necessary regions for the interaction depending on PAR. Ubiquitination of ALC1 by CHFR was dependent on PARylation and resulted in the degradation of PARylated ALC1. PARP1/2 inhibitors decreased the ubiquitination of PAR-dependent ALC1, and the expression of ALC1 was upregulated by PARP1/2 inhibitors. Ubiquitination mediated by CHFR resulted in the degradation of ALC1. In conclusion, PARP1/2 inhibitors decrease the ubiquitination of ALC1 leading to the accumulation of ALC1, which affects the therapeutic effects of DNA damage response drugs in breast cancer treatment.

\section{Introduction}

PARP inhibitors are toxic to cells with defects in homologous recombination (HR)-mediated DNA double-strand break (DSB) repair, including cells with mutations in BRCAI and $B R C A 2$, genes whose loss of function predisposes patients to breast and ovarian cancer (1). BRCA1 and BRCA2 are the most important genetic factors in hereditary breast cancer (2). PARP1/2 inhibitors induce synthetic lethality in cancer cells defective in the HR repair pathway including BRCA1/2 (3). Previous research indicates that PARP1 [poly(ADP-ribose) polymerase 1] facilitates DNA repair by binding to DNA breaks and attracting DNA repair proteins to the site of damage (4). Moreover, the local chromatin relaxation at DNA damage sites is regulated by PARP1 enzymatic activity (5). Many patients benefit from the treatment of PARP inhibitors (6). Furthermore, PARP inhibitor, niraparib, also showed significant clinical benefit in patients without HR deficiencies (7). Given the expanding clinical use of PARP inhibitors and the high likelihood of acquired resistance, there is a significant need to identify and overcome the mechanisms of resistance.

Amplified in liver cancer $1(A L C l)$ [also known as $C H D I L$ (chromodomain-helicase-DNA-binding protein 1-like], a poly(ADP-ribose) and ATP-dependent remodeler is involved in the chromatin-relaxation process $(8,9)$. The 
presence of ALC1 overexpression has been suggested to be associated with aggressive tumor biology in breast cancer, multiple myeloma and lung cancer (10-13). Additionally, ALC1 interacts with PARP1/PARylation in base excision repair (14). Notably, this interaction is mediated through the interplay of the ALC1 macro-domain and the PAR moiety of PARylated-PARP1 (15), which activates ALC1 at sites of DNA damage (16). CHFR (checkpoint with forehead-associated and RING finger domains) is a nuclear protein and functions as a tumor suppressor in the early mitotic checkpoint by actively delaying passage into mitosis in response to mitotic stress $(17,18)$. Previous research has demonstrated that CHFR functions as an E3 ubiquitin ligase, resulting in interaction between CHFR and PARP1 induced by mitotic stress (19). Additionally, the interaction between CHFR and PARP1 plays an important role in cell cycle regulation and cancer therapy (20). Given the evidence that primary and secondary resistance to PARP inhibition have led to treatment failure (21), the development of new biomarkers and the ability to identify potential mechanisms of resistance are vital. However, the associations among PARP, CHFR and ALC1 in regards to cancer development and therapeutic response remain undefined.

\section{Materials and methods}

Breast cancer tissues. A cohort of 28 paired human breast and peripheral non-tumor tissues extracted during surgical resection were collected from breast cancer patients in Shanghai Changhai Hospital from 2013 January to 2015 January. All cancer specimens were frozen in liquid nitrogen and stored at $-80^{\circ} \mathrm{C}$ after surgical resection. This study was approved by the Institutional Review Board of the Second Military Medical University (Shanghai, China). All participants gave informed consent before they entered the study. The patients included all women, with a median age of 54 years (range 31-85 years).

Quantitative Real-time PCR. Total RNA was extracted from breast cancer tissues or peripheral non-cancer tissues samples using the TRIzol Reagent (Invitrogen) according to the manufacturer's instructions, and 1-2 $\mu \mathrm{g}$ of RNA was treated by RNase-free DNaseI (Takara) to remove genomic DNA contamination. qRT-PCR analysis was conducted using a SYBR Green Supermix kit (Toyobo, Osaka, Japan) with a Light Cycler 480 II (Roche, Basel, Switzerland). The cycle parameters were $95^{\circ} \mathrm{C}$ for a 1 min hot start and 45 cycles of $95^{\circ} \mathrm{C}$ for $10 \mathrm{sec}, 60^{\circ} \mathrm{C}$ for $10 \mathrm{sec}$ and $72^{\circ} \mathrm{C}$ for $20 \mathrm{sec}$. The fold change in expression was calculated using the $\Delta \Delta \mathrm{Ct}$ method with the B2M mRNA as an internal control. Experiments for each sample were performed with two duplicates.

Cell lines and cell culture. The human breast carcinoma cell line (MCF-7), and 293T cells (CRL-3216) were obtained from the Shanghai Cell Bank of the Chinese Academy of Sciences (Shanghai, China). The cells were maintained in Dulbecco's modified Eagle's medium (DMEM) (Thermo Fisher Scientific, Inc.) and $1 \%$ penicillin/streptomycin $(\mathrm{P} / \mathrm{S})$ (Sigma-Aldrich; Merck KGaA) supplemented with $10 \%(\mathrm{v} / \mathrm{v})$ fetal bovine serum (FBS) (Pan). All cells were grown at $37^{\circ} \mathrm{C}$ with $5 \% \mathrm{CO}_{2}$ in a humidified incubator.
Plasmids. Full length UB (ubiquitin) was cloned into a modified pCDNA3 vector to generate encoding hemagglutinin (HA)-tagged UB. Meanwhile, ALC1 was cloned into the pRIES2-EGFP vector to construct S-FLAG-SBP (SFB)-tagged ALC1. The pCMV-Myc/HA-CHFR recombinant plasmid was constructed. The deletion mutants of CHFR were generated by using the QuikChange Site-Directed Mutagenesis kit (Stratagene; Agilent Technologies, Inc.) according to the manufacturer's protocol.

Antibodies, chemicals and reagents. Antibodies against ALC1 (cat. no. ab51324; Abcam), anti-HA (cat. no. ab18181; Abcam) and GAPDH (cat. no. ab8245; Abcam) were purchased from Abcam. Anti-FLAG (cat. no. F3165; Sigma), anti- $\beta$-actin (cat. no. A5441; Sigma), anti-Myc (cat. no. M4439; Sigma) antibodies were purchased from Sigma-Aldrich/Merck KGaA. Anti-CHFR antibody (cat. no. PA5-28079) was purchased from Thermo Fisher Scientific, Inc. The working concentration of antigens was 1:1,000 dilution.

MG132, dimethyl sulphoxide (DMSO) and cycloheximide (CHX) were purchased from Sigma. MG132 and CHX were prepared in DMSO to obtain 10 and $100 \mu \mathrm{M}$ stock solutions, respectively. Aliquots were stored at $-20^{\circ} \mathrm{C}$ to avoid freeze-thaw cycles, and a working solution was freshly prepared with culture medium immediately prior to use.

The PARP inhibitors AZD2281 (olaparib), and PJ34 (iniparib) were purchased from Selleck Chemicals. AZD2281 and PJ34 were dissolved in DMSO, and stored as per the manufacturers' recommendations. Cells were seeded at $1 \times 10^{6}$ cells in $10 \mathrm{ml}$ of medium and $24 \mathrm{~h}$ after seeding, the cells were treated with $10 \mu \mathrm{M}$ AZD2281 or $10 \mu \mathrm{M}$ PJ34 for $24 \mathrm{~h}$ in fresh medium.

Protein affinity purification. The soluble fraction was incubated with $0.5 \mathrm{ml}$ of streptavidin-conjugated beads (Thermo Fisher Scientific, Inc.) at $4^{\circ} \mathrm{C}$ for $2 \mathrm{~h}$. The beads were washed three times with NETN100 buffer. Associated proteins were eluted with $2 \mathrm{mM}$ biotin in $1 \mathrm{X}$ PBS and incubated further with $50 \mu \mathrm{l}$ of S beads (Novagen) at $4^{\circ} \mathrm{C}$ for an additional $2 \mathrm{~h}$, and then washed by NETN buffer five times. The bound proteins were eluted with SDS sample buffer (4\% SDS, $20 \%$ glycerol, $10 \%$ 2-mercaptoethanol, $0.004 \%$ bromphenol blue, $0.125 \mathrm{M}$ Tris-HCl), analyzed by SDS-PAGE (polyacrylamide gel electrophoresis) and silver staining. The objective band was analyzed by mass spectrometry.

Plasmid transfection. The 293T cells were seeded in 6-well plates $\left(4 \times 10^{5}\right.$ cells/well) one day before transfection. The next day, transfection was performed using Lipofectamine 2000 reagent (Thermo Fisher Scientific, Inc.) when the cells reached $\sim 80-90 \%$ confluence according to the manufacturer's protocol. Medium was replaced after 4-6 h with complete medium with FBS and P/S. Cells were harvested for use after $48 \mathrm{~h}$ of incubation.

Western blot analysis. The cells were harvested and washed with phosphate-buffered saline (PBS). Next, the cells were lysed with $30 \mathrm{ml}$ of ice-cold NETN100 buffer [150 mM $\mathrm{NaCl}, 1 \%$ Triton X-100, $1 \mathrm{mM}$ phenylmethyl-sulfonyl fluoride, and $25 \mathrm{mM}$ Tris ( $\mathrm{pH} 7.5$ )] containing cocktail which 


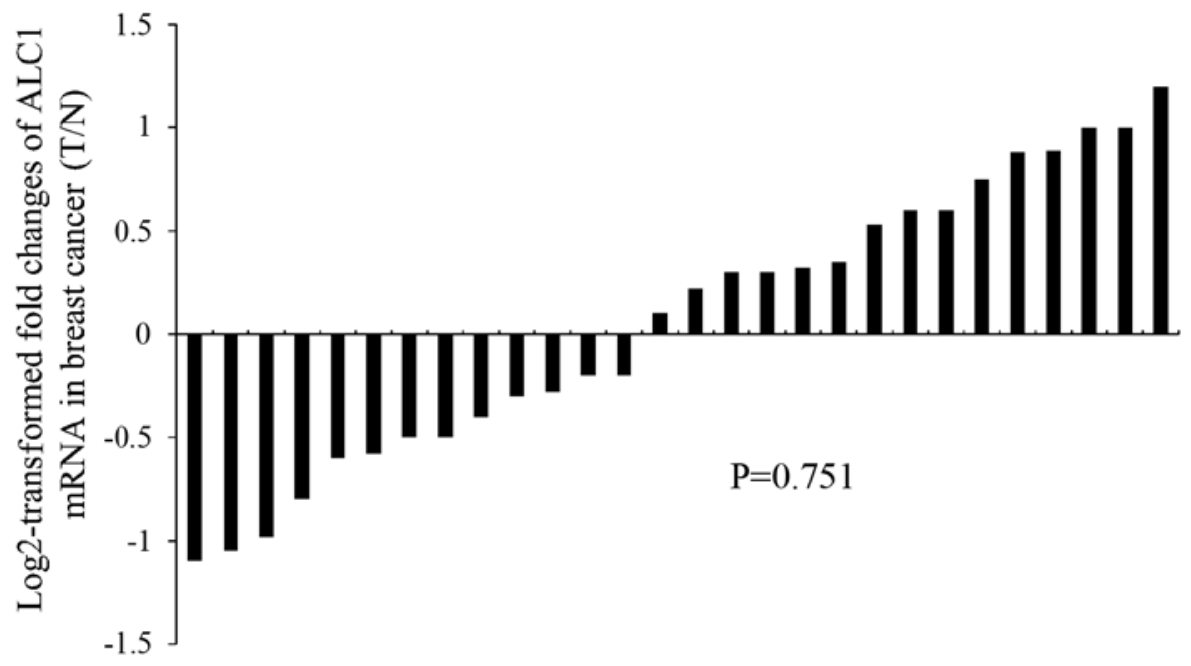

Figure 1. The mRNA level of $A L C 1$ was analyzed in 28 paired breast cancer tissues with their corresponding non-cancerous tissues by quantitative reverse transcriptase PCR (RT-qPCR). The columns show the upregulated or downregulated fold change of ALC1 mRNA level in breast cancer tissues, as compared with that in the corresponding adjacent non-cancerous liver, where $\beta$-actin was used as an internal control. ALCl, amplified in liver cancer protein 1.

was the protease inhibitor (Santa Cruz Biotechnology, Inc.). The cell lysates were centrifuged at $12,000 \mathrm{x} \mathrm{g}$ at $4^{\circ} \mathrm{C}$ for $8 \mathrm{~min}$. The clear supernatant extract was boiled in SDS buffer (SDS, glycerol, bromic acid, $1 \mathrm{M}$ Tris $\cdot \mathrm{HCl}$ ) for $8 \mathrm{~min}$ and stored at $-20^{\circ} \mathrm{C}$. A total of $100 \mu 1$ of the cell lysates were subjected to electrophoresis on SDS-12.5\% polyacrylamide gels. Then, the separated proteins were blotted on a PVDF (polyvinylidene fluoride) membrane (GE Healthcare) using a semi-dry transfer unit (Bio-Rad). The membranes were incubated in TBS with $5 \%$ non-fat milk and $0.1 \%$ Tween-20 for $1 \mathrm{~h}$ at room temperature (RT) and then incubated with the primary antibodies overnight at $4{ }^{\circ} \mathrm{C}$. After washing with Tris-buffered saline containing $0.1 \%$ Tween-20, the membranes were incubated with a secondary antibody, either HRP-conjugated anti-mouse IgG (Santa Cruz Biotechnology, Inc.; sc-516176, dilution 1:2,000) or HRP-conjugated anti-rabbit IgG (Santa Cruz Biotechnology, Inc.; cat. no. sc-516087; dilution 1:2,000) for $40 \mathrm{~min}$ at RT. The blots were visualized with a chemiluminescent ECL kit (Santa Cruz Biotechnology, Inc.).

Co-immunoprecipitation and immunoblotting. Cells were lysed with $1 \mathrm{X}$ cell lysis buffer (Cell Signaling Technology) and rotated at $12,000 \mathrm{x} \mathrm{g}$ at $4^{\circ} \mathrm{C}$ for $8 \mathrm{~min}$. Cell debris was removed by centrifugation and the soluble fraction was collected and precleared with protein $\mathrm{A} / \mathrm{G}$ agarose beads for $2 \mathrm{~h}$ at $4^{\circ} \mathrm{C}$. The precleared cell lysate was incubated with the indicated antibodies (anti-FLAG antibody) overnight followed by incubation with protein $\mathrm{A} / \mathrm{G}$ beads for at least $2 \mathrm{~h}$ at $4^{\circ} \mathrm{C}$. Immunoprecipitates were then washed 6 times with cell lysis buffer and boiled in $1 \mathrm{X}$ SDS loading buffer. Samples were resolved on SDS-PAGE and transferred to PVDF membranes and immunoblotting was carried out with antibodies as indicated.

Statistical analysis. The statistical analyses were performed with the ANOVA and a relevant post hoc test. A value of $\mathrm{P}<0.05$ was considered indicative of a statistically significant result. All data were analyzed using either GraphPad Prism 6 software (GraphPad Software, Inc.) or SPSS 20.0 (IBM). Values are shown as mean \pm SEM for each group.

\section{Results}

Transcription level of ALC1 is not regulated in breast cancer tissues. Although the presence of ALC1 overexpression has been observed in breast cancer tissues (10), little is known concerning the mRNA level of ALC1 in breast cancer development. To address this issue, we evaluated the transcriptional expression level of ALC1 in breast cancer tissues and peripheral non-tumor tissues. There was no difference in relative mRNA levels in 28 breast cancer samples compared with the adjacent non-cancerous tissues as analyzed by quantitative real-time PCR (qPCR) (Fig. 1).

CHFR interacts with ALC1. In order to investigate the high level of ALC1 in breast cancer, mass spectrometry analysis was used to analyze the associated proteins. Stably expressing SBP-tagged ALC1 293T cells were generated and used to identify the associated proteins of ALC1 by tandem affinity purification. Compared with the control group, a component with a molecular weight of $\sim 70 \mathrm{kDa}$ was observed in the purified complex by silver staining. Mass spectrometry analysis demonstrated that the band represented CHFR (Fig. 2A).

To confirm the interaction between CHFR and ALC1 in the physiological condition, we performed reciprocal immunoprecipitation of the exogenous proteins. SBP-ALC1 plasmids were transfected into the first group and HA-CHFR plasmids were transfected into the second group. SBP-ALC1 and HA-CHFR plasmids were co-transfected into the third group. Cell lysates were collected $30 \mathrm{~h}$ later. The samples were analyzed by western blot analysis. The protein bands on the PVDF membranes were detected with anti-FLAG antibody and anti-HA antibody. As a result, the bands of the interacting proteins were detected in the third group, and the interaction was not found in the first or second group. As expected, exogenously expressed HA-Tagged CHFR interacted with SBP-Tagged ALC1 in vitro (Fig. 2B). To 


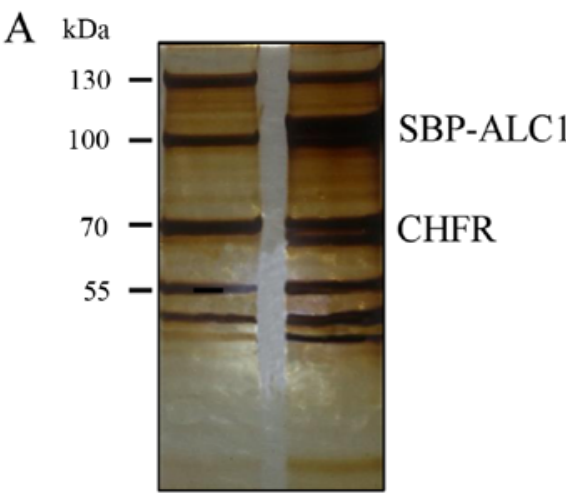

C
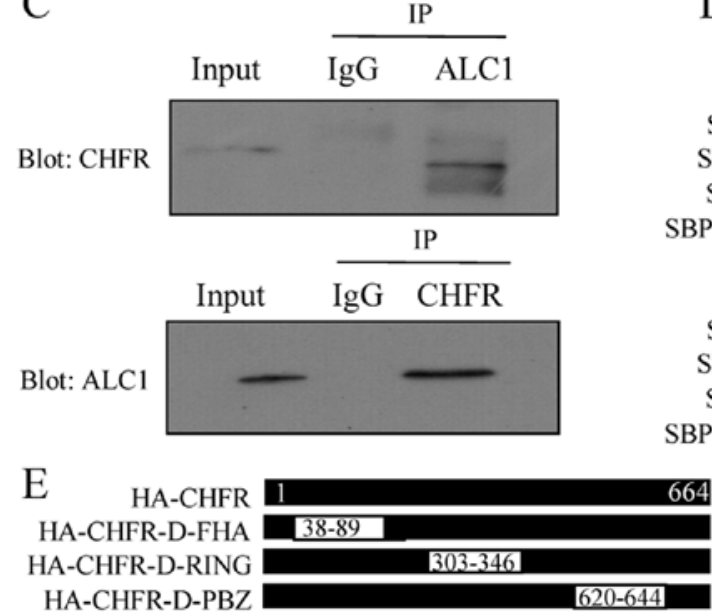

HA-CHFR-D-PBZ

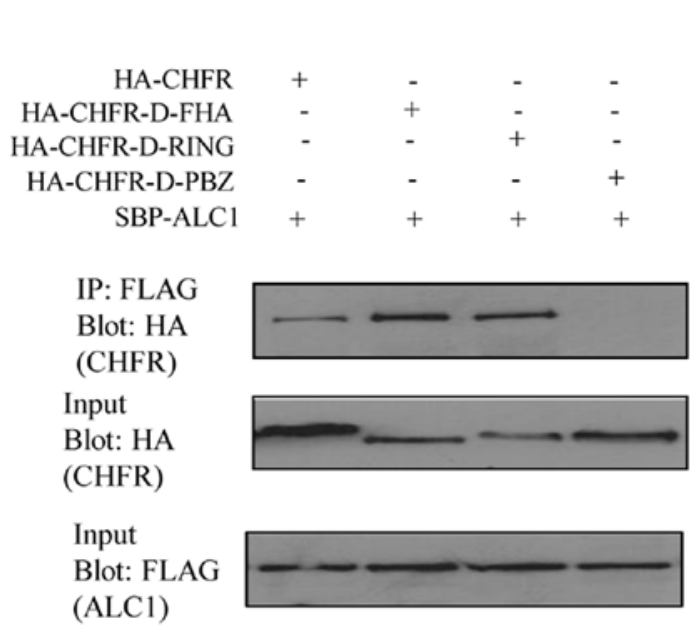

B

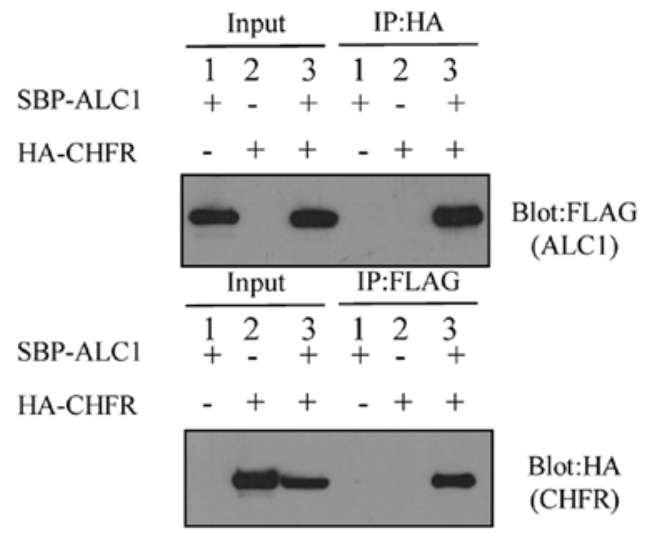

D

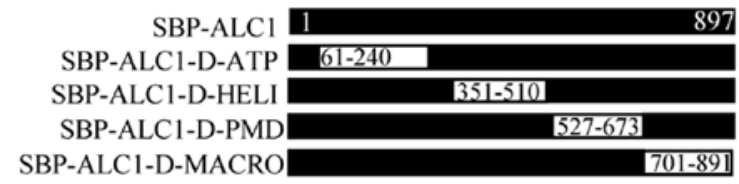

SBP-ALCl +

$\begin{array}{rccccc}\text { SBP-ALCl } & + & - & - & - & - \\ \text { SBP-ALC1-D-ATP } & - & + & - & - & - \\ \text { SBP-ALC1-D-HELI } & - & - & + & - & - \\ \text { SBP-ALC1-D-PMD } & - & - & - & + & - \\ \text { SBP-ALC1-D-MACRO } & - & - & - & - & + \\ 664 \text { HA-CHFR } & + & + & + & + & +\end{array}$

\section{IP: HA \\ Blot: FLAG \\ (ALC1)}

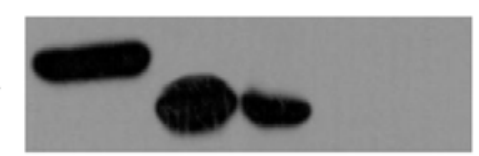

Input

Blot: FLAG

(ALC1)

Input

Blot: HA

(CHFR)

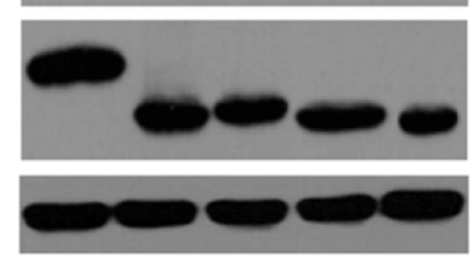

Figure 2. CHFR interacts with ALC1. (A) Silver staining of affinity-purified ALC1 complexes. The extracts of 293T cells stably expressing SBP-ALC1 or SBP vector were subjected to tandem affinity. The protein bands were analyzed by mass spectrometry. The bands were labeled as SBP-ALC1 and CHFR. (B) ALC1 interacts with CHFR in vitro. SBP-ALC1 and HA-CHFR were co-transfected into 293T cells. Cell lysates were analyzed by co-immunoprecipitation (IP) and western blotting with HA or Flag antibodies respectively. (C) ALC1 interacts with CHFR in vivo. MCF-7 cell lysates were analyzed by IP and western blotting with anti-ALC1 or CHFR antibodies, respectively. IgG was used as the control. (D) SBP-tagged wild-type ALC1 and deletion mutants were transfected with HA-CHFR into $293 \mathrm{~T}$ cells. Cell lysates were pulled down by streptavidin-conjugated beads and examined by western blotting using HA antibody. (E) HA-tagged CHFR and its deletion mutants were transfected with SBP-ALC1 into 293T cells. Cell lysates were immunoprecipitated with anti-FLAG antibody, and examined by western blotting using anti-HA antibody. CHFR, checkpoint with forehead-associated and RING finger domains; ALC1, amplified in liver cancer protein 1; CHD1L, chromodomain-helicase-DNA-binding protein 1-like.

explore whether endogenous ALC1 interacts with CHFR-like exogenous proteins, endogenous immunoprecipitation experiments were performed. MCF-7 cells were collected, some were incubated with CHFR antibody with NETN lysate, and the others were incubated with $\operatorname{IgG}$ as control.
Then the proteins were detected by western blot analysis. The PVDF membranes were incubated with the ALC1 antibody. The ALC1 band was found in the panel incubated with CHFR antibody previously, but no band was detected in the other panel (Fig. 2C). To map the ALC1-interacting 
A DMSO AZD2281 PJ34
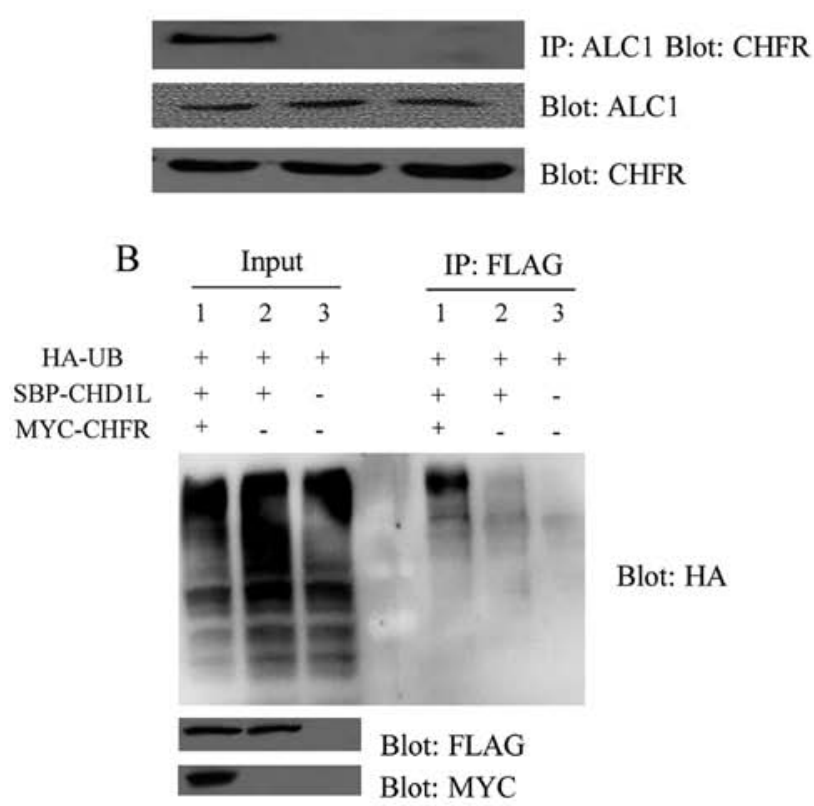

Blot: HA

\begin{tabular}{|c|c|c|c|c|c|c|}
\hline $\mathrm{C}$ & & $\mathrm{np}$ & & & $\mathrm{FI}$ & \\
\hline & 1 & 2 & 3 & 1 & 2 & 3 \\
\hline HA-UB & + & + & + & + & + & + \\
\hline SBP-CHD1L & + & + & + & + & + & \\
\hline MYC-CHFR & + & + & + & + & + & \\
\hline DMSO & + & - & - & + & - & \\
\hline AZD2281 & - & + & - & - & + & - \\
\hline PJ34 & - & - & + & - & - & \\
\hline
\end{tabular}

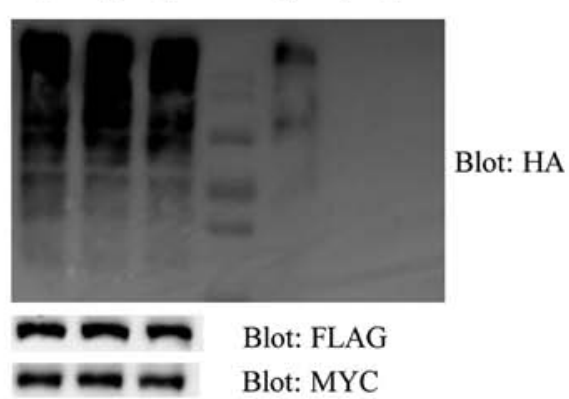

Figure 3. Ubiquitination (Ub) of ALC1 by CHFR is dependent on PARylation. (A) The endogenous ALC1 protein was unable to bind to CHFR with PARP. (B) UB bands were significantly deepened in the first panel compared with the other groups in 293T cells. (C) UB bands were observed in the first group, but not in the group with the addition of PARP1 inhibitors. PARP, poly(ADP-ribose) polymerase; CHFR, checkpoint with forehead-associated and RING finger domains; ALC1, amplified in liver cancer protein 1.

region with CHFR, ATP (adenosine triphosphate) (aa61-240) domain, Helicase (aa351-510) domain, PMD (PAR modification domain) (aa527-673) domain, and MACRO (aa701-891) domain deletion mutants of ALC1 were generated. Immunoprecipitation experiments showed that both the MACRO domain (376-592aa) and PMD (aa527-673) deletion mutants failed to associate with CHFR (Fig. 2D). Meanwhile, to map the CHFR-interacting region with ALC1, FHA (filamentous hemagglutinin) (aa38-89) domain, RING (aa303-346) domain and PBZ (poly(ADP-ribose)-binding zinc finger) (aa620-644) deletion mutants of CHFR were generated and their abilities to interact with ALC1 were tested. Only the PBZ deletion mutant failed to interact with ALC1 (Fig. 2E).

Ubiquitination of ALCl by CHFR is dependent on PARylation. We sought to determine whether PAR is crucial for the interaction of ALC1 and CHFR. The results showed that the endogenous ALC1 protein cannot bind to CHFR with PARP inhibitors which disrupt the PAR interaction. However, the endogenous ALC1 proteins were bound to CHFR without PARP inhibitors, suggesting that PARP1/2 inhibitors influence the interaction between ALC1 and CHFR proteins (Fig. 3A). PARP inhibitors were found to abolish the PAR-dependent recruitment of CHFR and ALC1 to the DSB sites. These data indicated that PAR deficiency could suppress the interaction between CHFR and ALC1. Yet, it was not ascertained whether CHFR regulates the level of $\mathrm{ALC1}$ protein. The CHFR contains a RING domain, which has the function of UB ligase E3. Since the E3 enzymes play the most important role in the ubiquitination reaction, we hypothesized that UB may be able to bind to the ALC1 through CHFR. ALC1 is ubiquitinated and degraded by proteasome through the ubiquitin-proteasome pathway, thereby expression of ALC1 is reduced. Based on this hypothesis, experiments were designed. First, to verify whether there is ubiquitination, three groups were used. HA-UB was transfected in the first group, HA-UB and SBP-ALC1 were transfected in the second group, HA-UB, SBP-ALC1 and MYC-CHFR were transfected in the third group. The experiments were performed using $293 \mathrm{~T}$ cells, respectively. After $24 \mathrm{~h}$ of transfection, MG132 at a concentration of $10 \mu \mathrm{M}$ was added to inhibit proteolysis and the cells were harvested after $4 \mathrm{~h}$. The cells were lysed with a NETN lysate containing $10 \mu \mathrm{M}$ MG132. The transferred PVDF membranes were incubated with HA, FLAG and MYC antibodies to evaluate the transfection effect. The results showed that UB bands were significantly attenuated in the third panel compared with the other groups in 293T cells. The results suggest that ALC1 can be ubiquitinated mediated by CHFR (Fig. 3B). Downregulation of the level of $\mathrm{ALC1}$ protein is related to the ubiquitination of itself. It is not known whether the regulation is affected by PARP inhibitors. To verify this issue, the following experiment was performed. HA-UB, SBP-ALC1 and MYC-CHFR plasmids were all transfected into the three groups of $293 \mathrm{~T}$ cells at the same time. To one group AZD2281was added, PJ34 was added to another group, and DMSO was added to the control group. After $24 \mathrm{~h}$ of transfection, MG132 was added at the concentration of $10 \mu \mathrm{M}$ to inhibit proteolysis, and the cells were harvested after $4 \mathrm{~h}$. UB bands were observed in the control group, but not in the group with the addition of PARP1/2 inhibitors (Fig. 3C). These results suggested that PARP1/2 inhibitors can inhibit the ubiquitination of ALC1.

CHFR regulates the stability of $A L C 1$. As a result of ubiquitination of ALC1 mediated by CHFR, the level of ALC1 protein may be decreased. The plasmids HA-CHFR-WT, HA-CHFR-D-RING and HA-CHFR-D-PBZ were transfected into 293T cells to express CHFR proteins respectively. The expression of CHFR was detected by HA antibody and the expression of ALC1 protein was detected by ALC1 antibody, and GAPDH was used as internal reference. The expression 

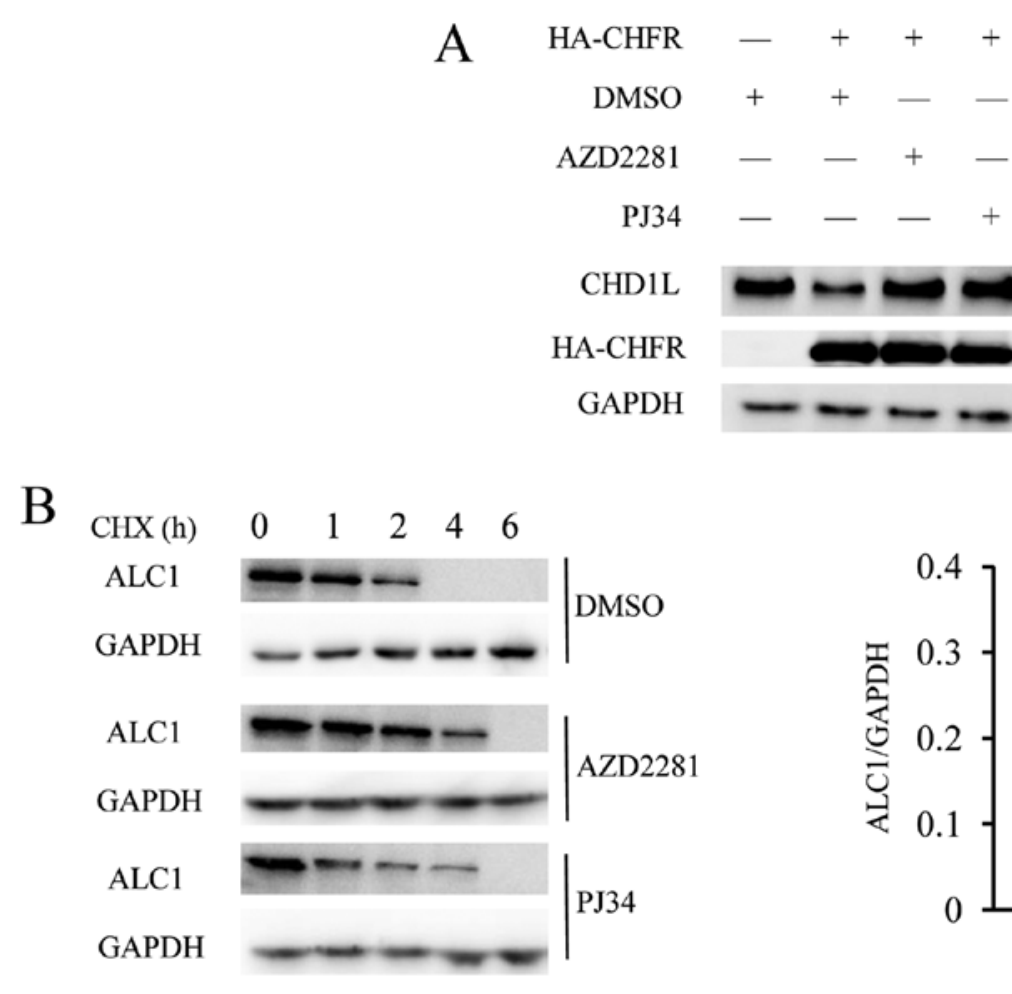

HD1L

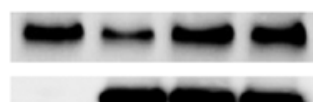

GAPDH
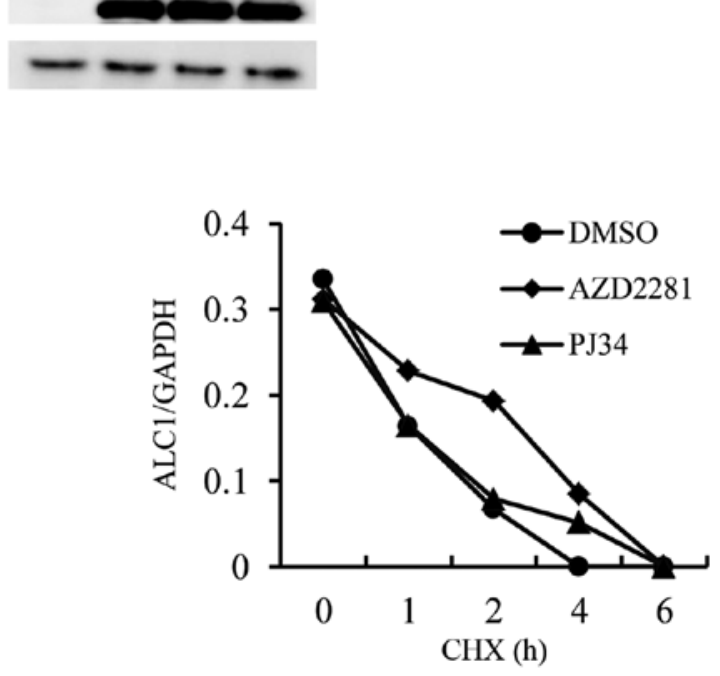

Figure 4. CHFR regulates the stability of ALC1. (A) CHFR overexpression decreased the protein level of ALC1 (CHD1L). The decrease in ALC1 by CHFR was reversed by PARP inhibitors. (B) The decrease in the half-life of ALC1 by CHFR was suppressed by PARP inhibitors. CHFR promoted ALC1 degradation. Three groups were treated with cycloheximide (CHX), the sample was collected at the indicated time points and then immunoblotted with antibodies against ALC1 and GAPDH. Quantification of ALC1 protein levels (normalized to GAPDH) is shown. PARP, poly(ADP-ribose) polymerase; CHFR, checkpoint with forehead-associated and RING finger domains; ALC1, amplified in liver cancer protein 1; CHD1L, chromodomain-helicase-DNA-binding protein 1-like.

of the endogenous ALC1 protein was decreased in cells with CHFR overexpression. To investigate the effect of PARP inhibitors on ubiquitination of ALC1, AZD22811 and PJ34 were added to the cells transfected with HA-CHFR. In addition, untransfected cells were used as negative controls, and transfected cells treated with DMSO were used as positive controls. The results showed that the expression of endogenous ALC1 was not decreased with the addition of PARP inhibitors, suggesting that PARP inhibitor could inhibit the ubiquitination of ALC1 mediated by CHFR (Fig. 4A).

To compare the expression of ALC1 protein in the presence and absence of PARP1/2 inhibitors, the effect of PARP1/2 inhibitors on ALC1 half-life was examined. The MCF-7 cells were cultured for $24 \mathrm{~h}$, and then the culture media were replaced with the addition of PARP1/2 inhibitors, AZD2281, PJ34 or DMSO at a concentration of $10 \mu \mathrm{M}$. After $10 \mathrm{~h}, \mathrm{CHX}$ was added at a final concentration of $100 \mu \mathrm{M}$. At $0,1,2,4$ and $6 \mathrm{~h}$, the cells were harvested. The results showed that the PARP1/2 inhibitors extended the half-life of ALC1, indicating that PARP1/2 inhibitors increased the expression of ALC1 (Fig. 4B).

\section{Discussion}

Local chromatin relaxation at DNA damage sites is regulated by PARP1 enzymatic activity which is one of the earliest cellular responses to DNA damage (22). PARP1/2 inhibitors lead to the inhibition of PARylation with various oncogenic proteins to inhibit the DNA repair pathway (23). Yet PARP inhibitor resistance is also a growing concern in the clinical setting. The most widely accepted mechanism of PARP1/2 inhibitor resistance is the restoration of the HR pathway through secondary reversion mutations (24). Moreover, a previous study showed that acquisition of PARP1/2 inhibitors and cisplatin resistance is associated with replication fork protection in BRCA2-deficient tumor cells (25). Our study revealed a new mechanism. ALC1, a poly(ADP-ribose)- and ATP-dependent remodeler, is involved in the chromatin-relaxation process regulated by PARP1 (8). Moreover, ALC1 is an oncogene located at Chrlq21 and it is amplified in many solid tumors (26). ALC1 is highly expressed in breast cancer tissues, and high expression of ALC1 protein suggests poor prognosis (13). The relationship among drug resistance, high expression of ALC1 and PARP inhibition remains unclear. Furthermore, the mRNA of ALC1 is not regulated in breast cancer tissues. The results indicated that there was no significant difference in the transcription level of ALC1 between breast cancer tissues and adjacent non-cancerous tissues. It was speculated that epigenetic modifiers regulate ALC1 expression. Then we further analyzed the associated proteins interacting with ALC1. CHFR was found to bind with ALC1 by mass spectrometry. CHFR functions as an E3 Ub-ligase of associated proteins and is responsible for its proteasome degradation (27). The results indicate that CHFR may play a crucial role in the regulation of ALC1.

Based on the results, we speculated how the ALC1 protein interacts with the CHFR protein and a schematic representation of the interaction is shown (Fig. 5). First, PARP1/2 binds 


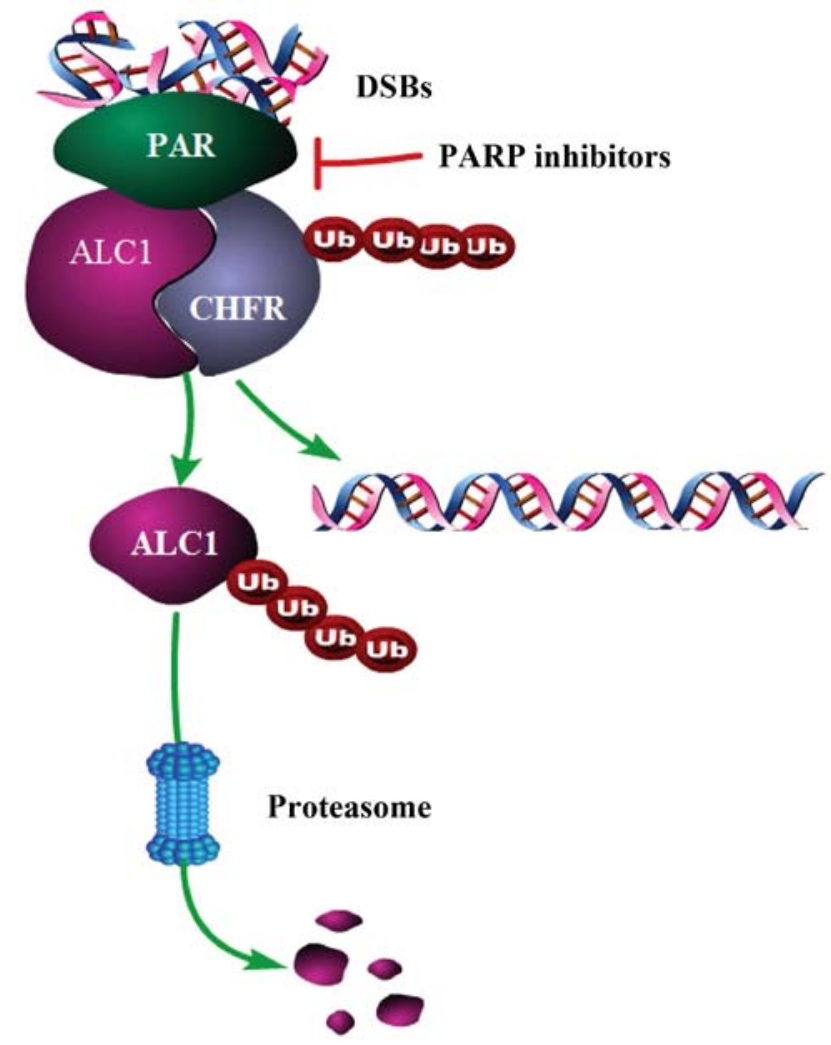

Figure 5. Schematic representation of the interaction between ALC1 and CHFR. CHFR, checkpoint with forehead-associated and RING finger domains; ALC1, amplified in liver cancer protein 1; Ub, ubiquitination; PARP, poly(ADP-ribose) polymerase; DSBs, double strand breaks.

rapidly to the site of DNA double-strand breaks (DSBs) and undergoes PARylation when the DNA is damaged. Next, ALC1 and CHFR are recruited to the site of DNA damage. The macro domain of ALC1 binds to PAR, and then CHFR can recognize ALC1 through the PBZ domain. It was found that CHFR could mediate the ubiquitination of ALC1 and affect the stability of ALC1 protein, which could lead to the degradation of ALC1. The presence of CHFR can keep ALC1 from playing a role only in the DNA damage response, but not in mediating malignant biological behavior. In the treatment of breast cancer, the use of PARP inhibitors may inhibit PARylation. Then ALC1 and CHFR would not be recruited together at the sites of DSBs. Thus, ubiquitination of ALC1 would be reduced and accumulation of ALC1 would be higher than before. Once ALC1 protein is highly expressed, its potential malignant biological behavior may affect the prognosis of the patient. For most BRCA1/2-deficient patients, high expression of ALC1 has no impact on the therapeutic effect of PARP1/2 inhibitors. However, for patients who exhibit resistance to PARP1/2 inhibitors, high ALC1 should not be ignored. This study also elucidated the reason why the expression of ALC1 protein is high in breast cancer, but the mRNA level is normal. This is a type of epigenetic modification. In brief, PARP1/2 inhibitors may turn on another tumor proliferation pathway while shutting down DNA damage repair, which may be one of the causes for the failure of the efficacy of PARP inhibitors. ALC1 is recruited by PARP1/2 at DNA damage sites, and degraded by CHFR. However, PARP1/2 inhibitors can reverse the degradation of ALC1 by
CHFR. High ALC1 accumulation contributes to development of cancer. In other words, PARP1/2 inhibitors, DNA damage drugs combined with anti-ALC1 drugs may help to improve the therapeutic effect for breast cancer patients. This should be considered when choosing therapeutic strategies for patients. By inhibiting ALC1 expression, PARP1 inhibitors can be effective for more breast cancer patients that exhibit potential drug resistance. However, one limitation of the study is that PARP1/2 gene knockout cell lines were not constructed, which can validate the expression of ALC1 without PARP1/2.

To conclude, in the present study, we demonstrated that ALC1 can interact with CHFR depending on PAR. Ubiquitination mediated by CHFR functioning as an E3 ubiquitin ligase results in the degradation of ALC1. PARP1/2 inhibitors decrease the ubiquitination of ALC1 and lead to the accumulation of ALC1, which affects the therapeutic effects of DNA damage response drugs in breast cancer treatment.

\section{Acknowledgements}

We thank Yongjun Dang of the School of Basic Medical Science, Fudan University for the fruitful scientific discussion and the sharing of various reagents.

\section{Funding}

This study was supported by the National Natural Science Foundation of China (81572591).

\section{Availability of data and materials}

All data generated or analyzed during this study are included in this published article.

\section{Authors' contributions}

YaW and JW conceived and designed the experiments. YinW and YirW performed the experiments. YirW, YinW and FL collected and analyzed the data. LY and CS contributed to acquisition of the reagents/materials/analysis tools and interpretation of data for the study. YirW and NW wrote the manuscript and JW and NW revised the manuscript. All authors read and approved the manuscript and agree to be accountable for all aspects of the research in ensuring that the accuracy or integrity of any part of the work are appropriately investigated and resolved.

\section{Ethics approval and consent to participate}

This study was approved by the Institutional Review Board of the Second Military Medical University (Shanghai, China). All participants gave informed consent before they entered the study.

\section{Patient consent for publication}

Not applicable.

\section{Competing interests}

The authors declare that they have no competing interests. 


\section{References}

1. Kolb AL, Gunn AR and Lakin ND: Redundancy between nucleases required for homologous recombination promotes PARP inhibitor resistance in the eukaryotic model organism Dictyostelium. Nucleic Acids Res 45: 10056-10067, 2017.

2. Sie AS, Spruijt L, van Zelst-Stams WA, Mensenkamp AR, Ligtenberg MJ, Brunner HG, Prins JB and Hoogerbrugge N High satisfaction and low distress in breast cancer patients one year after BRCA-mutation testing without prior Face-to-Face genetic counseling. J Genet Couns 25: 504-514, 2016.

3. Drew Y, Ledermann J, Hall G, Rea D, Glasspool R, Highley M, Jayson G, Sludden J, Murray J, Jamieson D, et al: Phase 2 multicentre trial investigating intermittent and continuous dosing schedules of the poly(ADP-ribose) polymerase inhibitor rucaparib in germline BRCA mutation carriers with advanced ovarian and breast cancer. Br J Cancer 114: 723-730, 2016.

4. Bryant HE, Schultz N, Thomas HD, Parker KM, Flower D, Lopez E, Kyle S, Meuth M, Curtin NJ and Helleday T: Specific killing of BRCA2-deficient tumours with inhibitors of poly(ADP-ribose) polymerase. Nature 434: 913-917, 2005.

5. Kulkarni A, Oza J, Yao M, Sohail H, Ginjala V, Tomas-Loba A, Horejsi Z, Tan AR, Boulton SJ and Ganesan S: Tripartite Motif-containing 33 (TRIM33) protein functions in the poly(ADP-ribose) polymerase (PARP)-dependent DNA damage response through interaction with amplified in liver cancer 1 (ALC1) protein. J Biol Chem 288: 32357-32369, 2013.

6. Rugo HS, Olopade OI, DeMichele A, Yau C, van't Veer LJ, Buxton MB,Hogarth M, Hylton NM,Paoloni M,Perlmutter J, et al: Adaptive randomization of veliparib-carboplatin treatment in breast cancer. N Engl J Med 375: 23-34, 2016.

7. Mirza MR, Monk BJ,Herrstedt J, Oza AM, Mahner S, Redondo A, Fabbro M, Ledermann JA, Lorusso D, Vergote I, et al: Niraparib maintenance therapy in Platinum-sensitive, recurrent ovarian cancer. N Engl J Med 375: 2154-2164, 2016.

8. Sellou H, Lebeaupin T, Chapuis C, Smith R, Hegele A, Singh HR, Kozlowski M, Bultmann S, Ladurner AG, Timinszky G, et al: The poly(ADP-ribose)-dependent chromatin remodeler Alc1 induces local chromatin relaxation upon DNA damage. Mol Bio Cell 27: 3791-3799, 2016.

9. Ahel D, Horejsi Z, Wiechens N, Polo SE, Garcia-Wilson E, Ahel I, Flynn H, Skehel M, West SC, Jackson SP, et al: Poly(ADP-ribose)-dependent regulation of DNA repair by the chromatin remodeling enzyme ALC1. Science 325: 1240-1243, 2009.

10. Wu J, Zong Y, Fei X, Chen X, Huang O, He J, Chen W, Li Y, Shen K and Zhu L: Presence of CHD1L over-expression is associated with aggressive tumor biology and is a novel prognostic biomarker for patient survival in human breast cancer. PLoS One 9: e98673, 2014.

11. Xu X, He Y, Miao X, Wu Y, Han J, Wang Q, Liu J, Zhong F, $\mathrm{Ou} \mathrm{Y}$, Wang Y and He S: Cell adhesion induces overexpression of chromodomain helicase/ATPase DNA binding protein 1-like gene (CHD1L) and contributes to cell adhesion-mediated drug resistance (CAM-DR) in multiple myeloma cells. Leuk Res 47: 54-62, 2016.

12. He LR, Ma NF, Chen JW, Li BK, Guan XY, Liu MZ and Xie D: Overexpression of CHD1L is positively associated with metastasis of lung adenocarcinoma and predicts patients poor survival. Oncotarget 6: 31181-31190, 2015.

13. Mu QJ, Li HL, Yao Y,Liu SC, Yin CG and Ma XZ: Chromodomain Helicase/ATPase DNA-binding protein 1-Like Gene (CHD1L) expression and implications for invasion and metastasis of breast cancer. PLoS One 10: e0143030, 2015.
14. Tsuda M, Cho K, Ooka M, Shimizu N, Watanabe R, Yasui A, Nakazawa Y, Ogi T, Harada H, Agama K, et al: ALC1/CHD1L, a chromatin-remodeling enzyme, is required for efficient base excision repair. PLoS One 12: e0188320, 2017.

15. Jiang BH, Chen WY, Li HY, Chien Y, Chang WC, Hsieh PC, Wu P, Chen CY, Song HY, Chien CS, et al: CHD1L regulated PARP1-driven pluripotency and chromatin remodeling during the early-stage cell reprogramming. Stem Cells 33: 2961-2972, 2015.

16. Lehmann LC, Hewitt G, Aibara S, Leitner A, Marklund E, Maslen SL, Maturi V, Chen Y, van der Spoel D, Skehel JM, et al: Mechanistic insights into autoinhibition of the oncogenic chromatin remodeler ALC1. Mol Cell 68: 847-859.e7, 2017.

17. Sun Z, Liu J, Jing H, Dong SX and Wu J: The diagnostic and prognostic value of CHFR hypermethylation in colorectal cancer, a meta-analysis and literature review. Oncotarget 8: 89142-89148, 2017.

18. Privette LM, Gonzalez ME, Ding L, Kleer CG and Petty EM: Altered expression of the early mitotic checkpoint protein, CHFR, in breast cancers: Implications for tumor suppression. Cancer Res 67: 6064-6074, 2007.

19. Kashima L, Idogawa M, Mita H, Shitashige M, Yamada T, Ogi K, Suzuki H, Toyota M, Ariga H, Sasaki Y and Tokino T: CHFR protein regulates mitotic checkpoint by targeting PARP-1 protein for ubiquitination and degradation. J Biol Chem 287: 12975-12984, 2012.

20. Brodie SA, Li G, Donald H, Khuri FR, Vertino PM and Brandes JC: Small molecule inhibition of the CHFR-PARP1 interaction as novel approach to overcome intrinsic taxane resistance in cancer. Oncotarget 6: 30773-30786, 2015.

21. Lim JS and Tan DS: Understanding resistance mechanisms and expanding the therapeutic utility of PARP inhibitors. Cancers (Basel) 9: E109, 2017.

22. Luijsterburg MS, de Krijger I, Wiegant WW, Shah RG, Smeenk G, de Groot AJ, Pines A, Vertegaal AC, Jacobs JJ, Shah GM and van Attikum H: PARP1 Links CHD2-mediated chromatin expansion and H3.3 deposition to DNA Repair by Non-homologous End-Joining. Molecular Cell 61: 547-562, 2016.

23. Rajawat J, Shukla N and Mishra DP: Therapeutic targeting of Poly(ADP-Ribose) Polymerase-1 (PARP1) in cancer: Current developments, therapeutic strategies, and future opportunities. Med Res Rev 37: 1461-1491, 2017.

24. Barber LJ, Sandhu S, Chen L, Campbell J, Kozarewa I, Fenwick K, Assiotis I, Rodrigues DN, Reis Filho JS, Moreno V, et al: Secondary mutations in BRCA2 associated with clinical resistance to a PARP inhibitor. J Pathol 229: 422-429, 2013.

25. Wang YT, Yuan B, Chen HD, Xu L, Tian YN, Zhang A, He JX and Miao ZH: Acquired resistance of PTEN-deficient cells to PARP inhibitor and Ara-C mediated by 53BP1 loss and SAMHD1 overexpression. Cancer Sci 109: 821-831, 2018.

26. Singh HR, Nardozza AP, Möller IR, Knobloch G, Kistemaker HA, Hassler M, Harrer N, Blessing C, Eustermann S, Kotthoff C, et al: A Poly-ADP-Ribose trigger releases the Auto-inhibition of a chromatin remodeling oncogene. Mol Cell 68: 860-871.e7, 2017.

27. Kim M, Kwon YE, Song JO, Bae SJ and Seol JH: CHFR negatively regulates SIRT1 activity upon oxidative stress. Sci Rep 6: 37578, 2016. 